Wolfgang Zuse

\title{
Akzeptanz von Gewalt und Sympathielenkung bei Macbeth: Erfahrungen aus Unterricht und Schule
}

\section{Einleitung}

Sympathielenkung ist kein etablierter Begriff, jedoch ein literarisches Phänomen, das in ganz unterschiedlichen Textsorten feststellbar, als künstlerisches Mittel wirksam wird und rezeptionsästhetisch fassbar ist. Natürlich haben sich auch Literaturwissenschaftler mit diesem Thema näher befasst, wie z.B. Manfred Pfister, der in seinem Aufsatz „Zur Theorie der Sympathielenkung im Drama“ (1978) eine Begriffsklärung vorgenommen hat, die meines Erachtens bei Behandlung von Texten unter diesem Aspekt eine hilfreiche Orientierung darstellt.

Sympathielenkung versteht er auf zwei Ebenen: Sie betrifft zum einen die ästhetische Einstellung des Publikums den fiktiven Figuren und Geschehnisabläufen gegenüber, die zwischen den Polen der Identifikation und des Engagements und einer Neutralen oder kritischen Distanz variiert und zum anderen die ganzheitliche, gefühlsmäßige, moralisch wertende und intellektuelle Momente integrierende 
Reaktion des Publikums auf die Dramenfiguren, die sich in ein Spektrum von uneingeschränkter Sympathie bis zu uneingeschränkter Antipathie abstufen lässt.

Aus einem Symposium zu dem Thema „Sympathielenkung in den Dramen Shakespeares" (1978) sind weitere Arbeiten hervorgegangen, von denen hier nur die eine von Ina Schabert „Sympatby als rezeptionsästhetische Kategorie in der englischen und amerikanischen Shakespearekritik" genannt werden soll, weil die Autorin Anglisten sicher gut bekannt ist und ganz gewiss denen, die der Deutschen Shakespeare-Gesellschaft angehören.

Dieser Vortrag basiert im wesentlichen auf Erfahrungen aus dem Literaturunterricht, es sind jedoch auch die Kenntnisse der genannten Autoren zur theoretischen Absicherung der praktisch erworbenen Einsichten berücksichtigt worden.

Bevor es aber um Macbeth geht, sollen zwei Beispiele aus ganz anderen literarischen Bereichen eingefügt werden, weil sie initiierend dazu geführt haben, dem Phänomen der Sympathielenkung näher nachzuspüren. Es sind Beispiele, die nachweisen, dass Schüler (hier zur sprachlichen vereinfachung hinfort als Kollektivbegriff verwandt) unter bestimmten Konstellationen in Texten dargestellte Verbrechen affektiv wie kognitiv zu entschuldigen oder sogar zu rechtfertigen bereit sind.

In einem Englischbuch für 10. Klassen (Learning English - Modern Course Gym 6) ist eine als „The Moose and the Sparrow" betitelte Geschichte enthalten, in der es darum geht, daß sich in einem Holzfällercamp in Kanada ein sehr schmächtiger 19-jähriger Student als Hilfskraft verdingt hat und von einem sehr großen, starken und brutalen Holzfäller, genannt Moose, verhöhnt, gequält und terrorisiert wird.

Als ihm niemand helfen kann, greift er zur Selbsthilfe, indem er unmittelbar vor einer tiefen Schlucht einen in der Nacht unsichtbaren Draht zwischen zwei Bäume spannt, um den vom Kartenspielen und einem Saufgelage heimkehrenden Moose zum Abstürzen zu bringen. Das Vorhaben gelingt: der Holzfäller wird am nächsten Morgen tot in der Schlucht aufgefunden, ohne dass bei irgendjemandem der Gedanke an einen Mordanschlag aufkommt, also sich auch kein Verdacht gegen den Verursacher des augenscheinlichen Unfalls richtet.

Bei der Erörterung des Ausgangs dieser Geschichte und bei der Frage, wie der Student Cecil zu beurteilen oder zu verurteilen sei, waren die Schüler sich weitgehend einig, dass diesem ja nichts anderes als diese Art der Selbsthilfe übrig geblieben sei und dass sie sein Entkommen ohne Mordanklage billigten. Der menschliche Aspekt blieb hier undifferenziert einseitig.

Die Sympathielenkung wird in dieser Geschichte zum einen von dem DavidGoliath-Paradigma bestimmt, zum anderen von den Qualen, die der Schmächtige und Hilflose auszustehen hat und für den bei den Schülern ein starkes Mitgefühl, aber auch das Verständnis für seine Rache aufkam. Die Struktur der Geschichte beeinflusst in nicht unerheblichem Maße die sympathetische Einstellung: der Handlungsverlauf ist ganz auf das Verhalten der Personen konzentriert, die Ab- 
sicht Cecils nur ganz unauffällig angedeutet, und der Mord wird erst im Schlusssatz erwähnt.

Es handelt sich hier um eine einfache Geschichte, aber entscheidende Kriterien der Sympathielenkung sind deutlich erkennbar: sie gehen aus der Handlungsentwicklung hervor, sind personenbezogen und erfahren durch Kontrastierung besondere Intensität. Dass Sympathielenkung hier eine Rechtfertigung für den Mord bewirkte, war Grund genug für eine korrigierende Erörterung.

Was in dieser Geschichte durchaus komplex angelegt ist, erscheint in Hemingways Roman A Farewell To Arms als eher punktuelle Sympathielenkung. Frederic Henry, Protagonist und Ich-Erzähler des Romans, braucht als verantwortlicher Offizier einer Ambulanz während eines eiligen Rückzugs im Kampfgeschehen des Ersten Weltkrieges Hilfe beim Schieben des stecken gebliebenen Krankenwagens. Er befiehlt zwei vorbeieilenden Sergeants dabei zu helfen. Als diese sich mit der Bemerkung, er sei nicht ihr Offizier und könne ihnen keine Befehle erteilen, weigern und weiter laufen, erschießt er den einen und verfehlt nur knapp den anderen, den er auch hatte töten wollen.

Sein Untergebener erbittet daraufhin die Waffe, um auf den am Boden Liegenden noch einmal schießen zu können. Als die Pistole versagt, gibt Lt. Henry technische Hinweise, woraufhin der Untergebene auf den Sergeant zwei Kopfschüsse abgibt und diesen dann an den Beinen wegzieht. Bei der Rückgabe der Pistole gibt er zu erkennen, dass er auf dieses Töten stolz sei, und Lt. Henry fragt interessiert nach, ob er selbst den zweiten Sergeant wohl auch noch erwischt habe. Äußerlich sehr anrüchig, werden dann noch Mantel und Umhang des Toten als Hilfsmittel zur Beweglichmachung der Ambulanz benutzt.

Über diese sehr brutale und menschenverachtende Szene wurde im Leistungskurs eines 12. Jahrgangs zunächst hinweg gelesen. In der Besprechung wurde dann deutlich, dass die Schüler sich von dem mit positiven Merkmalen gekennzeichneten Protagonisten hatten lenken lassen. Was der Offizier in der Verantwortung einer Ambulanz und in einer Kriegssituation tut, erschien ihnen darüber hinaus richtig und vertretbar. Dass es sich jedoch hier um einen Mord und um ein geradezu lustvolles Töten handelt, musste erst noch geklärt werden.

Nimmt man mögliche Wirkungen von Literatur ernst und will man verantwortungsbewusst Literaturdidaktik betreiben, dann bedarf es klärender Gespräche, damit dargestellte Verbrechen weder übergangen noch zu rechtfertigen oder zu entschuldigen versucht werden. Dies erscheint umso dringender geboten, als nicht auszuschließen ist, dass Medien wie Film und Fernsehen undifferenziertes Denken und Verhalten fördern. 


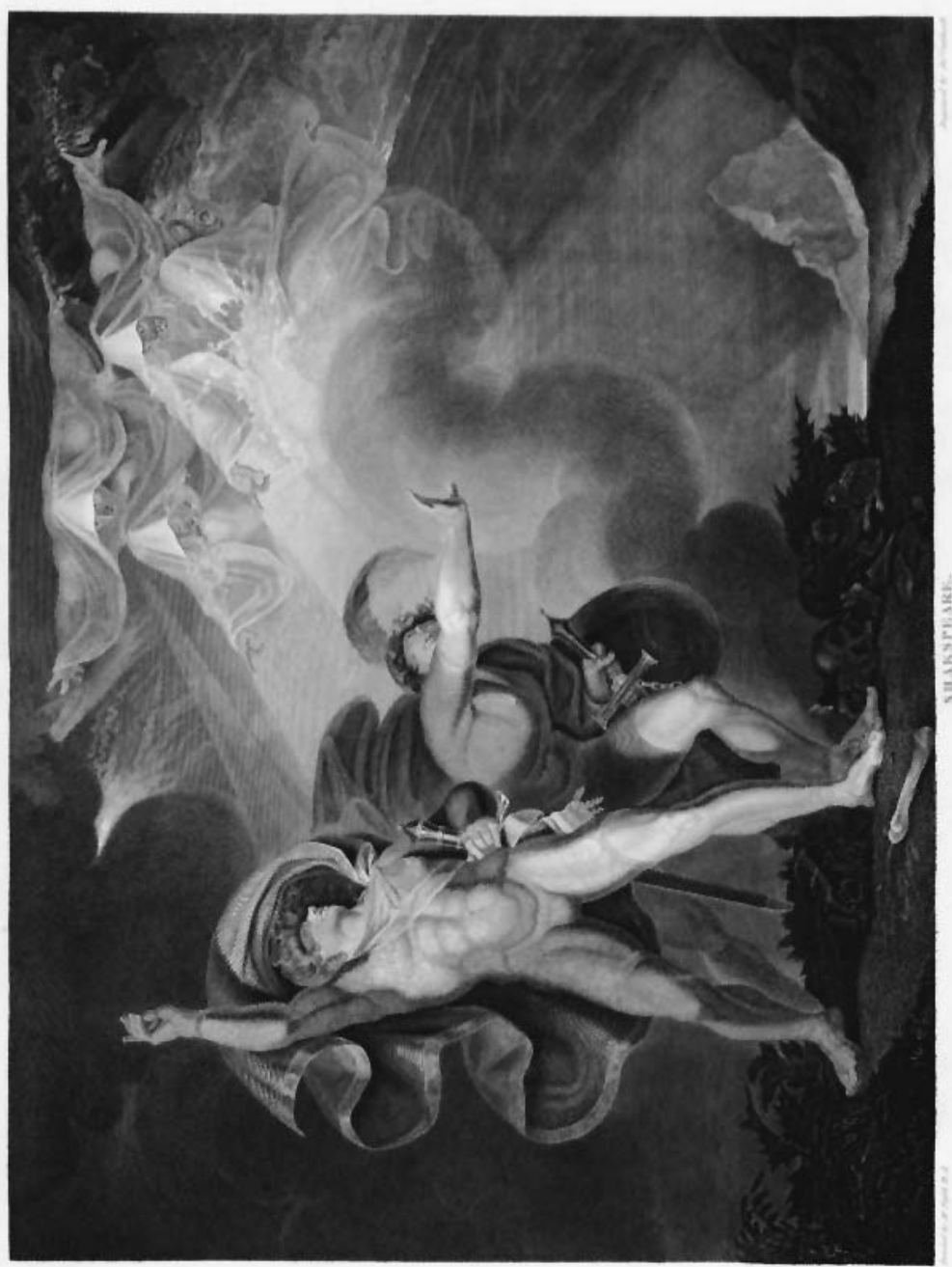

Abb. 11: J. H. Füssli, Macbeth (Act I, Scene 3): A Heath. Macbeth, Banquo and Three Witches 


\section{Sympathielenkung in Macbeth}

Und nun zu Macbeth: Die Boydell-Ausstellung präsentiert sieben Bilder zu Shakespeares Drama, und Johann Heinrich Füssli hat Macbeth auf zwei Bildern in Gestalt und Pose nach Art eines antiken Helden dargestellt. Mit Bezug auf Akt I, Szene 3 - Macbeth und Banquo begegnen den Hexen auf der Heide - ist Macbeth hier nackt und mit ausgeprägter Muskulatur im Bild. Er trägt ein Schwert, und hinter seinem Rücken wird ein Umhang in großen Falten sichtbar. Mit hoch gestrecktem rechtem Arm ist er den Hexen zugewandt, während Banquos rechter Arm eher als Abwehr zu verstehen ist.

Nach heutiger Begrifflichkeit, und geht man vom rein Faktischen aus, dann ist Macbeth als Massenmörder zu bezeichnen. Er begeht drei Morde eigenhändig, König Duncan und seine zwei Diener; er lässt seinen Kampfgefährten und Vertrauten Banquo umbringen, dessen Sohn entfliehen kann. Auf seinen Befehl hin werden die Frau, die Kinder und der gesamte Anhang seines Widersachers Macduff umgebracht, und er selbst tötet leichthin nach kurzem Kampf den ihm weit unterlegenen jungen Siward.

Offenbar in ihrer Entscheidung auch ,sympathiegelenkt', haben die Herausgeber des Katalogs zur Boydell-Sammlung in Zusammenarbeit mit der Deutschen Shakespeare-Gesellschaft von all den hier ausgestellten Bildern ausgerechnet den von Füssli dargestellten Macbeth für das Deckblatt des Katalogs zur Shakespeare Gallery gewählt. Ebenso stilisiert erscheint Macbeth in Füsslis Bild ,The Witches show Macbeth the Descendants of Banquo' (Pape/Burwick 1996: 62). Sein hoch erhobener Arm weist hier die Nachkommen Banquos zurück, jedoch ist die Pose nicht angstvoll, sondern wiederum ,heldisch'.

Nur eines von Füsslis Bildern fügt sich treffend in das Thema dieses Vortrags ein. Es ist die sehr expressive Darstellung David Garricks als Macbeth und Lady Pritchards als Lady Macbeth in der Szene nach der Ermordung Duncans (Pape/Burwick 1996: 61). In charakteristischer Weise drückt sich im Gesicht Macbeths (Garricks) das blanke Entsetzen über den an seinem König begangenen Mord aus, während Lady Macbeth (Lady Pritchard) mit ausgestrecktem Arm nach den zwei blutigen Dolchen greifen will und mit kühler Geste den linken Zeigefinger erhoben hält. Sowohl auf diese Szene wie auf die unterschiedlichen Charakteristika und Verhaltensweisen von Macbeth und Lady Macbeth wird im Verlauf des Vortrags noch einzugehen sein. Wie nun diese Untaten von Rezipienten aufgenommen und wie Shakespeares ,Kunst der Sympathielenkung ${ }^{6}$ zu beeinflussen vermag, das soll anhand des Textes zu erklären versucht werden.

Der in unserer Literaturdidaktik enthaltene Anspruch, dass die Subjektivität des Interpretierenden zur Geltung kommen soll, impliziert, dass Schüler mit ihren Erfahrungen, Anschauungen und Gefühlen um das Verstehen von Handlung und Figuren bemüht sind. Diese rezeptionsästhetische Zugangsweise zum jeweils lite- 
rarischen Gegenstand bedeutet aber auch, dass die Charaktere eher als reale Menschen denn als Kunstfiguren erachtet und Problemstellungen nach eigenen lebensweltlichen Kriterien verstanden und behandelt werden.

So können spontane Eindrücke leicht zur Identifikation mit den Charakteren führen, an denen Schüler Merkmale, Eigenschaften und Verhaltensweisen erkennen, die ihnen vertraut sind. Sie lassen sich auch nicht nur durch positive Merkmale einer Figur leiten, sondern identifizieren sich mit Schwächen, Versuchungen, Ängsten, Leiden, Einsamkeit und Resignation - kurz mit allem, was sich in der sehr komplex gestalteten Figur Macbeth ausdrückt. So ist seine von Gut und Böse gekennzeichnete Menschlichkeit auch für junge Menschen attraktiv geblieben.

Er ist vieler Verbrechen schuldig und wird doch nie, wie allenthalben zu lesen ist, zu einem wirklich ,unsympathischen' Scheusal. Wie das möglich ist, soll im Folgenden einer näheren Betrachtung unterzogen werden, jedoch auch für den Unterricht pragmatische Konsequenzen anführen.

Macbeth ist eingangs als erfolgreicher Kämpfer in der Schlacht gegen die Rebellen, vom König dafür ausgezeichnet, eine mit positiven Charakteristika ausgestattete Figur, und als solche nimmt sie für sich ein. Macbeths Ehrgeiz ist zugleich seine Schwäche; er ist daher nur allzu leicht verführbar und erliegt der Versuchung, mehr zu wollen als ihm bestimmt ist. Lady Macbeth dagegen erzeugt krasse Abneigung, wenn sie, mit den Schwächen ihres Mannes vertraut, diese bloßstellt und ausnutzt. Das Gespräch über den zu planenden Mord an König Duncan ist gleichzeitig eine Kontrastierung der beiden Hauptgestalten, und diese wirkt sich zugunsten Macbeths aus. Seiner Güte, Schwäche und Unzuverlässigkeit - aus ihrer Sicht, stellt sich ihre Entschlossenheit, ihre Skrupellosigkeit und Grausamkeit als deutlicher Gegensatz gegenüber. Während Macbeth vor dem Königsmord zurückschreckt, ist sie unerbittlich, provoziert und demütigt ihn, vor allem dadurch, dass sie ihm Feigheit vorwirft und Zweifel an seiner Männlichkeit äußert.

So gewiss wie ihr antipathetische Gefühle entgegengebracht werden, so sicher wird Macbeth, der unwillig ist, den Mord zu begehen, jedoch unter dem Zwang psychologischer Verführung steht, Mitgefühl zuteil. Intendierte Wirkung und reale Reaktion auf den Text stimmen hier fraglos überein. Die Identifikation der Schüler mit Macbeth ist umso leichter möglich, als der geplante Mord an Duncan verbal weitgehend gemieden wird und es Shakespeare offensichtlich vor allem auf das Verhalten der beiden Hauptgestalten ankommt. In seinem ersten Monolog (es gibt insgesamt 24) gibt Macbeth Einblick in sein Denken und Fühlen vor dem Mord an Duncan. Er ist sich des Bösen der zu begehenden Tat bewusst und wägt das Für und Wider ab.

Er kommt hierher, zwiefach geschirmt; - zuerst,

Weil ich sein Vetter bin und Untertan,

Beides hemmt stark die Tat; dann, ich - sein Wirt,

Der gegen seinen Mörder schließen müßte

Das Tor, nicht selbst das Messer führen. - 
Dann hat auch dieser Duncan seine Würde

So mild getragen, blieb im großen Amt

So rein, daß seine Tugenden, wie Engel,

Posaunenzüngig, werden Rache schrein

Dem tiefen Höllengreuel seines Mordes;

Und Mitleid, wie ein nacktes, neugebornes Kind,

Auf Sturmwind reitend, oder Himmels Cherubim,

$\mathrm{Zu}$ Roß auf unsichtbaren, luft'gen Rennern,

Blasen die Schreckenstat in jedes Auge,

Bis Tränen Flut den Wind ertränkt. -

Ich habe keinen Stachel,

Die Seiten meines Wollens anzuspornen,

Als einzig Ehrgeiz, der, zum Aufschwung eilend,

Sich überspringt und jenseits niederfällt. -92

Der Mord als Vorhaben ist auch hier eher umschrieben als direkt benannt; Macbeths Reflektionen kreisen ausdrücklich und in Bildern um das, was für ihn gegen den Mord spricht, mit dem Ergebnis, dass sein einziges Motiv („,vaulting ambition“, übersteigerter Ehrgeiz) keinen Erfolg bringen wird. Folgerichtig will er Abstand nehmen („We will proceed no further in this business.“ I, 7, 31), ist aber dann doch nicht stark genug, sich gegenüber Lady Macbeth zu behaupten. Sie hat mit anderen, psychologisch radikaleren Mitteln fortgesetzt, was die Hexen bei Macbeth nur indirekt initiiert haben.

Das Ergebnis von Macbeths Disposition zum Bösen und seinem schwachen Willen wird als doppelte Verführung erkannt, so dass er bereits in diesem Stadium als Opfer zum Täter wird. Hier ist einzufügen, dass in einem pre-reading-Verfahren die Schüler auf witchcraft und Geistererscheinungen in elisabethanischer Zeit vorbereitet wurden, um dann den Versuch eines Transfers auf die heutige Zeit zu unternehmen. Genannt wurden beispielsweise Satanskulte mit verderblichen Einflüssen und Verführungen zum Bösen, Drogenkonsum mit persönlichkeitsverändernden Auswirkungen und Halluzinationen als Projektionen innerer Wünsche und Konflikte.

Dass die Ausführung der Tat im Hintergrund bzw. außerhalb des Bühnengeschehens bleibt und Macbeth bei seiner Rückkehr nur euphemistisch feststellt: „I have done the deed“ (II, 2, 16), verharmlost zwar den Mord nicht, erspart den

92 „He's here in double trust: / First, as I am his kinsman and his subject, / Strong both against the deed; then, as his host, / Who should against his murderer shut the door, / Not bear the knife myself. Besides, this Duncan / Hath borne his faculties so meek, hath been / So clear in his great office, that his virtues / Will plead like angels, trumpet-tongued against / The deep damnation of his taking-off. / And pity, like a naked newborn babe / Striding the blast, or heaven's cherubin horsed / Upon the sightless couriers of the air, / Shall blow the horrid deed in every eye, / That tears shall drown the wind. I have no spur / To prick the sides of my intent, but only / Vaulting ambition which oerleaps itself / And falls on th'other -“ (I, 7, 12-28) 
Rezipienten jedoch die Vergegenwärtigung der Brutalität des Geschehens und mäßigt ihre Abscheu. In Polanskis Verfilmung von Macbeth wird der Mord an Duncan beispielsweise in aller Ausführlichkeit gezeigt und nicht nur als ,Ergebnis festgestellt. Während also der Mord nur kurz erwähnt wird, sind die Folgen und Auswirkungen auf Macbeth umso ausführlicher vermittelt, und hierauf kam es Shakespeare ganz offensichtlich an. Es gelingt ihm dabei auch, teilnehmende Aufmerksamkeit ganz auf die Nachwirkungen der bösen Tat zu lenken, wie sie auf Macbeths Gewissen lastet und sich psychisch-physisch auf ihn auswirkt.

Seine Furcht vor der eigenen Schlaflosigkeit, das Entsetzen vor seinen schuldigen Händen, von denen er meint, sie würden ihm die Augen ausreißen und seien vom Blut, das Meere rot färben könne, nicht mehr zu reinigen (II, 3, 59 f.), sind so suggestiv, dass sie von Schülern, wie in Spontanphasen erkennbar, als seine Bestrafung empfunden wurden. Sie konnten sich jedoch auch mit seinen Leiden den Leiden eines Mörders - identifizieren. Das Mitgefühl mit Macbeth ist umso wahrscheinlicher als seine bewegende Reue und seine glaubhaften Gewissensqualen mit Lady Macbeths nüchterner, um nicht zu sagen kaltblütiger Reaktion auf den Tod Duncans, kontrastiert werden. Hier kommt Füsslis Gemälde mit David Garrick als Macbeth und Mrs Pritchard als Lady Macbeth erneut in Erinnerung. Sie wird die betäubten Kammerdiener durch das auf ihnen verschmierte Blut Duncans in Verdacht bringen und rät Macbeth, über die Tat nicht weiter nachzudenken: „A little water clears us of this deed/How easy is it then!“ (II, 3, $24 \mathrm{f}$.)

Wird bei der unmitteibaren Reaktion Macbeth noch relativ viel Mitgefühl zuteil, so wird ihm durch die Ermordung der zwei Kammerdiener Duncans auch wieder Sympathie entzogen. Seine Erklärung, warum er diese zwei getötet hat, lässt nun einen anderen Macbeth erkennen - einen Macbeth, der, um von seinem eigenen Verbrechen abzulenken, skrupellos zwei Morde begeht und in der Erklärung seines Motivs geschickt und heuchlerisch vorgibt, die Mörder aus Liebe zu Duncan getötet zu haben (II, 3, 109-119).

Die hier entstandene Distanzierung verstärkt sich eher noch, wenn Macbeth den Mordauftrag zur Tötung seines Kampf- und Weggefährten Banquo und seines Sohnes Fleance erteilt. Und wenn er dann klagt, „O full of scorpions is my mind, dear wife", dann leidet er keine Gewissensqualen, sondern er wird gequält von dem Gedanken an die Hexenprophezeiungen für Banquo. Zur Erinnerung: während Macbeth die Königswürde prophezeit wurde, sind es Banquos Nachkommen, die Könige werden.

Auch Macbeths Horrorerlebnis bei dem zweimaligen Erscheinen von Banquos Geist während des Banketts hat sich im Hinblick auf Sympathielenkung als nicht so eindeutig erwiesen, wie dies angesichts der von ihm erlittenen Qualen zu erwarten gewesen wäre. Zu unmittelbar folgen die Mitteilung an Macbeth, dass Banquo tot sei und dessen geisterhafte Erscheinung als rächende und strafende Instanz aufeinander, als dass dies nicht als gerecht empfunden werden könnte. 
Der König wird nicht zur Rechenschaft gezogen, aber er erfährt die Strafe für seinen Mordauftrag auf diese Weise. Lady Macbeths Versuche, gegenüber den Lords Erklärungen abzugeben und Macbeth zu ermahnen, männliche Haltung zu bewahren („Are you a man?“, III, 4, 59 f.), tragen noch dazu bei, dass Rezipienten die Wirkung der Geistererscheinung auf Macbeth weniger emotional bewegt als distanziert betrachten.

Schwankungen - Abschwächungen wie Intensivierungen der Sympathielenkung - sind jedoch als Shakespeares Gestaltungskunst in Handlungsentwicklung wie in Charaktergestaltung eingebunden und textintendiert.

So ist ein stärkeres Engagement für Macbeth wieder möglich, wenn er sich Hilfe suchend zu den Hexen begibt und ihre zweideutigen Prophezeiungen erfährt: Niemanden fürchten zu müssen, der von einer Frau geboren wurde, und unbesiegt zu bleiben, so lange nicht der Wald von Birnam sich auf sein Schloss Dunsinane zu bewege. Durch dramatische Ironie ist hier der Rezipient involviert, weil er weiß, dass Macbeth in die Irre geführt und erneut Opfer böser Mächte wird. Als am Ende dieser recht aufwendig gestalteten Szene Macbeth erfährt, dass Macduff nach England geflohen ist, ist sein kurz gehaltenes aside, mit der Ungeheuerlichkeit, die Familie Macduffs nebst Anhang auslöschen zu wollen, geeignet, ihm die kurz zuvor gewährte Anteilnahme gänzlich zu entziehen und ihn der Verdammung preiszugeben. Ein Wechsel zwischen Verzeihen und Anklage intensiviert jedoch in charakteristischer Weise die Identifikation mit Macbeth.

Nach Macbeths Hexenbefragung im IV. Akt ist eine episodische Handlungsstruktur bis zum V. Akt auffällig. In allen Episoden geht es bei Abwesenheit von Macbeth um ihn als Verräter, als Tyrannen, um den Mörder, um „devilish Macbeth“. Selbst die schlafwandelnde Lady Macbeth erinnert an seine und gemeinsam mit ihm begangenen Missetaten. Es geht zudem um Macbeths Vernichtung, die, wie auch Rezipienten wissen, als Vergeltung für seine Verbrechen und auch um weiteres Unheil abzuwenden, gerechtfertigt und notwendig wäre. Jedoch - es gelingt Shakespeare erneut, sobald Macbeth wieder präsent ist $(V .3,75)$, ihn so darzustellen, dass ihm Anteilnahme nur schwer zu versagen ist. Er wiegt sich in der vermeintlichen Sicherheit der Hexenvorhersagen und ist dabei doch ein Getäuschter; er ist einsam und von seinen Lords verlassen worden, zudem wird ihm mitgeteilt, dass 10.000 englische Soldaten gegen ihn versammelt sind. Seaton, sein Diener, ist ihm als einziger Gesprächspartner geblieben.

Seyton - mir wird speischlecht.

Seh ich so - Seyton sag ich! - dieser Sturm

Stößt mich zum Thron auf ewig oder jetzt vom Stuhl.

Ich hab genug gelebt; mein Lebensweg

Führt in die Dürre, unters gelbe Laub;

Und was den Altersherbst begleiten sollte,

Wie Ehre, Liebe, Freundeskreis, Respekt,

Danach darf ich nicht ausschaun; ernt anstatt 
Flüche, nicht laut, doch tief, und Lippendienst, Wind, dem das arme Herz gern weigern würde,. Und wagt's nicht! - - - -93

Wieder gewährt Macbeth hier Einblick in seine innere Situation; sie ist gekennzeichnet von unverstellter Offenheit und pessimistischer Zukunftserwartung. Die fast ohne Bilder unverschlüsselt geäußerten Eingeständnisse sind unschwer nachvollziehbar und bewegend, weil sie menschlich so eingängig sind. In diesem Monolog verdrängen andererseits Macbeths Visionen und das menschlich Anrührende an seiner Situation aber auch sein Wissen um eigenes Verschulden an seinem seelisch so zerrütteten Zustand.

Eine mögliche Vermutung, dass die so unabweisbare Sympathielenkung in Macbeth dennoch durch Zufall entstanden sein könnte, lässt sich unschwer durch den Verweis auf Shakespeares Richard III., den Schurken nach eigenem Bekunden, entkräften. In diesem Stück gibt es nur eine einzige Stelle, an der der Protagonist Mitgefühl erfahren kann, nämlich dann, wenn ihm die Geister der durch ihn Umgekommenen im Traum erschienen sind und er in unverstellter Weiser klagt:

\section{Schuldig! Schuldig!}

Ich muß verzweifeln. - Kein Geschöpfe liebt mich,

Und sterb ich, wird sich keine Seel erbarmen,

$\mathrm{Ja}$, warum solltens andre? Find ich selbst

In mir doch kein Erbarmen mit mir selbst. ${ }^{94}$

Schuldeingeständnisse, Einsamkeit, versagte Liebe und fehlendes Mitgefühl ermöglichen auch hier beim Zuschauer bzw. Leser emotionale Anteilnahme. Insgesamt aber bleibt es bei dieser Ausnahme.

Die überwiegend affektive Zuwendung zu Macbeth wird, und zwar deutlich sympathiegelenkt, bis zum Schluss aufrechterhalten. Seine resignative Einstellung zum Leben, existenziell tief empfunden und entsprechend metaphernreich in seinem Monolog (dem wohl bekanntesten überhaupt!) ausgedrückt, nachdem er vom Tode seiner Frau erfahren hat, kennzeichnen ihn in einer Situation, die schon tragische Merkmale enthält.

93 „Seyton! - I am sick at heart, / When I behold - Seyton, I say! - this push / Will cheer me ever or disseat me now. / I have lived long enough. My way of life / Is fall'n into the sere, the yellow leaf, / And that which should accompany old age, / As honour, love, obedience, troops of friends, / I must not look to have; but in their stead, / Curses, not loud but deep, mouthhonour, breath / Which the poor heart would fain deny, and dare not.“ (V, 3, 19-28)

94 „,...] Guilty! Guilty / I shall despair. There is no creature loves me; / And, if I die, no soul shall pity me: / Nay, wherefore should they, since that I myself / Find in myself no pity to myself?" $(\mathrm{V}, 3,119-203)$ 
SEYTON. Die Köngin, Herr, ist tot.

MACBETH. Sie hätte später sterben können; - es hätte

Die Zeit sich für ein solches Wort gefunden. -

Morgen, und morgen, und dann wieder morgen,

Kriecht so mit kleinem Schritt von Tag zu Tag,

Zur letzten Silb' auf unserm Lebensblatt;

Und alle unsre Gestern führten Narrn

Den Pfad des stäub'gen Tods. - Aus! kleines Licht! -

Leben ist nur ein wandelnd Schattenbild;

Ein armer Komödiant, der spreizt und knirscht

Sein Stündchen auf der Bühn', und dann nicht mehr

Vernommen wird; ein Märchen ist's, erzählt

Von einem Blöden, voller Klang und Wut,

Das nichts bedeutet ... ${ }^{95}$

Dem Einsamen und Schwächeren, der hier zum Opfer übermächtiger Gegner und der Täuschung durch übernatürliche Kräfte zu werden droht, der kann der emotionalen Zuwendung, zumal bei jungen Menschen als Rezipienten sicher sein.

\section{Sympathielenkung und (fiktionale) Rezipientenreaktion}

Aber auch Erwachsene sind vor derartiger emotionaler Vereinnahmung nicht gefeit, wie uns Willy Russel in seinem Theaterstück Educating Rita vermittelt, in dem er seine Protagonistin Rita nicht für Macduff, sondern für Macbeth Partei ergreifen lässt. Rita ist eine Friseuse, die sich an einer sogenannten Offenen Universität im Norden Englands für literarische Studien eingeschrieben hat. Nach ihren allerersten Theaterbesuch läuft sie über alle Maßen beeindruckt und aufgeregt zu ihrem Tutor, um diesem von dem Ereignis, dass sie Macbeth auf der Bühne gesehen habe, zu berichten: „War seine Frau nicht eine Kuh, eh? Und dann die fantastische Stelle, wo er Macduff trifft und denkt, dass er ganz unbesiegbar sei. Ich saß am Rande meines Sitzes an der Stelle. Ich wollte rufen und Macbeth Bescheid sagen, wollte ihn warnen. “96

95 „SEYTON. The queen, my lord, is dead. / MACBETH. She should have died hereafter; / There would have been a time for such a word. / Tomorrow, and tomorrow, and tomorrow / Creeps in this petty pace from day to day / To the last syllable of recorded time; / And all our yesterdays have lighted fools / The way to dusty death. Out, out, brief candle, / Lifes but a walking shadow, a poor player / That struts and frets his hour upon the stage / And then is heard no more. It is a tale / Told by an idiot, full of sound and fury / Signifying nothing." (V, 5, 16-28)

96 „Wasn't his wife a cow, eh? An' that fantastic bit where he meets Macduff an’ he thinks he's all invincible. I was on the edge of me seat at that bit. I wanted to shout out an' tell Macbeth, warn him.“ (45) 
Rita verhält sich hier ausschließlich gefühlsbetont (wie wohl so manch ein unvoreingenommener Abonnementsbesucher im Theater auch) und ist beispielhaft sympathiegelenkt. Ginge es nämlich um eine distanziert-kritische Einstellung bei der Konfrontation zwischen Macbeth und Macduff, so müssten Leser wie Zuschauer von Anfang an auf den Sieg Macduffs hoffen, der doch zwingende Veranlassung hat, aus persönlichen wie politischen Gründen den zu töten, der so viel Unheil angerichtet hat. Aber da bei Rezipienten das affektive Erleben stärker entwickelt worden ist, liegt auch die mitfühlende Parteinahme für Macbeth nahe. Die Eindrücke des Augenblicks überlagern die Schandtaten Macbeths, um nicht zu sagen, sie machen sie vergessen. (Über den Eindrücken der Gegenwart die Vergangenheit zu vergessen, ist doch bis heute aktuell und manchmal sogar brisant.)

\section{Sympathielenkung und Inszenierung}

Wie weit Sympathielenkung auch bei Inszenierungen von Macbeth dramaturgische Entscheidungen zu beeinflussen vermag, soll die filmische Aufzeichnung einer Inszenierung der Lincoln Center Theatre Company zeigen. In dieser wird sogar gegen den Text inszeniert, denn vor dem entscheidenden Kampf mit Macduff sagt Macbeth ausdrücklich, er werde nicht wie der „Roman fool“ (der römische Narr) durch das eigene Schwert sterben, sondern bis zum Letzten kämpfen. Das tut er dann auch, bis ihm von Macduff der Kopf abgeschlagen wird.

Sehr zur Überraschung des Textkundigen stirbt Macbeth in der Inszenierung nun doch wie ein Römer, wenn auch nicht durch das eigene Schwert: er wirft sich vielmehr in das Schwert Macduffs (vgl. Abb. 12-14). Indem er sich bekreuzigt, stirbt er augenscheinlich als Christ, was zu ihm, der nicht einmal Reue für seine Schandtaten zeigt, nicht recht passen will. Ebenso verwunderlich ist Macduffs verhalten, der Macbeth eine gewisse Anerkennung zuteil werden lässt, indem er ihm seine eigene Kette mit Kreuz übergibt (Abb. 15) und durch das Schlagen des Kreuzes über dem Toten diesem eine Art te absolvo gewährt (Abb. 16).

Zurück zum Text: Erst wenn Malcolm, der Sohn Duncans als proklamierter König seine zukunftsorientierte Rede hält und dabei an ,this dead butcher and his fiend-like queen" $(V, 7,69)$ erinnert, geht die intendierte Wirkung dahin, dass auch beim Publikum das nur affektive Erleben einer nachdenklichen Rückbesinnung weichen soll.

Wie mit Schülern ausführlich besprochen, erfüllt Shakespeare mit diesem Ende Bedingungen des Elisabethanischen Weltbildes, in dem, wer sich aus der göttlich determinierten Chain of Being (der großen Seinskette) ausklinkt und Chaos verursacht, wie Macbeth and Lady Macbeth, bestraft werden muss. Danach kann mit der Einsetzung Malcolms als König, die Ordnung in Schottland wieder hergestellt werden. 
Abb. 12-16: Szenenfotos aus der Macbeth-Inszenierung der Lincoln Center Theatre Company (Act V, Scene 8)

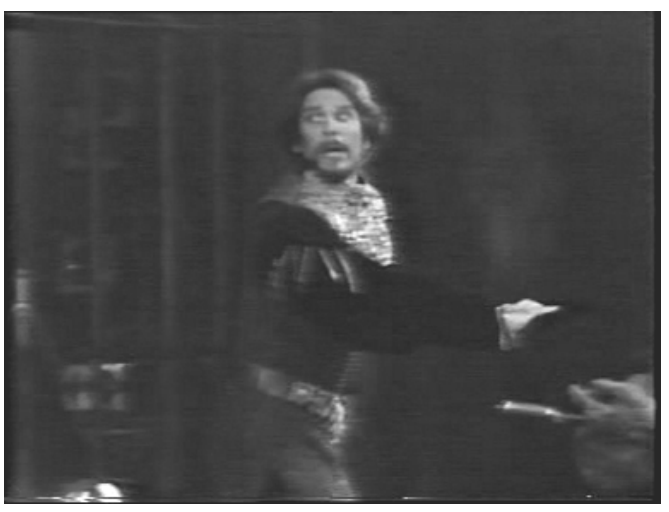

Abb. 12: Macbeth greift nach seinem Schwert, ohne es zu ziehen

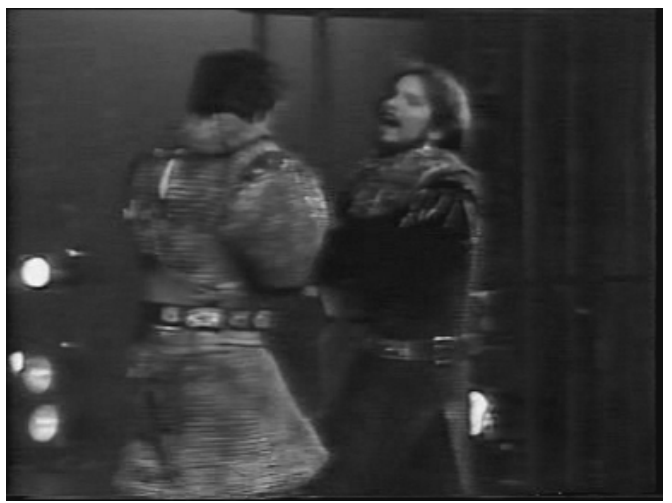

Abb. 13: Macbeth dreht sich zu Macduff und scheint ihn anzugreifen, wobei er in Macduffs Schwert läuft

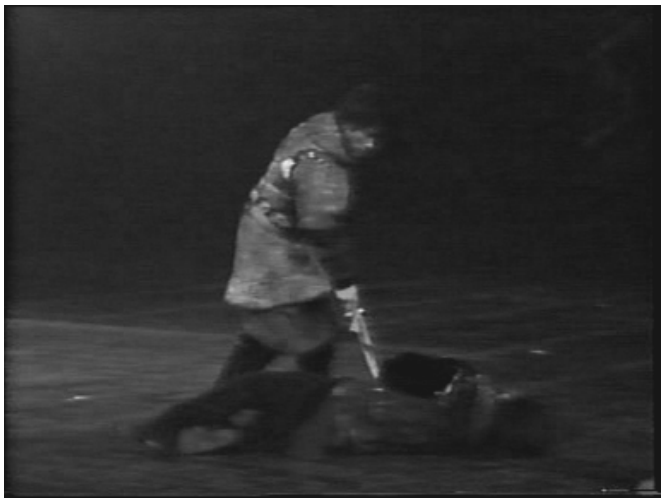

Abb. 14: Macbeth liegt am Boden; Macduff zieht sein Schwert zurück 


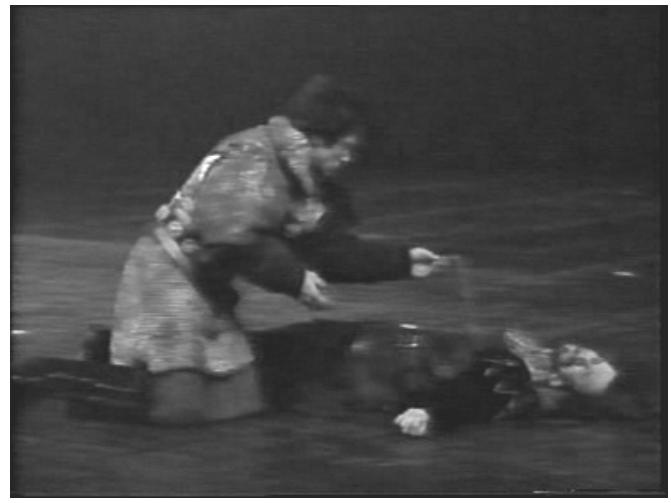

Abb. 15: Macduff hat sein Kreuz abgenommen und legt es Macbeth auf die Brust

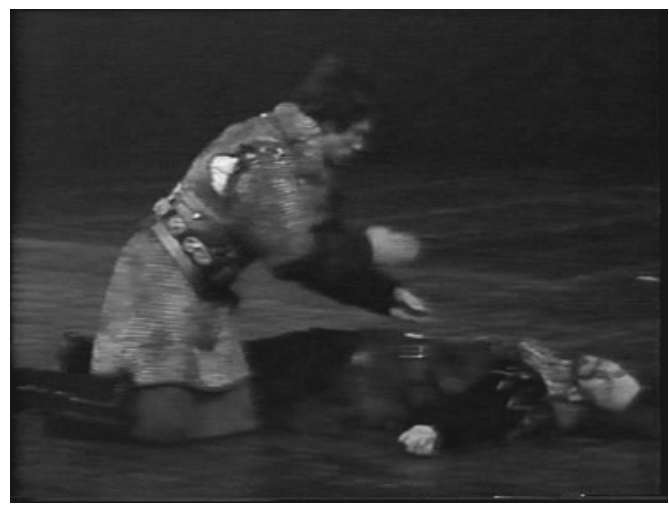

Abb. 16: Macduff schlägt das

Kreuz über Macbeth 
Als Tragödie erfüllte Macbeth nur bedingt die Kriterien der aristotelischen Tragikvorstellung. Macbeth ist zwar anfänglich der gefeierte Held einer siegreichen Schlacht - und soweit wären Füsslis ,heldische ${ }^{6}$ Macbeth-Darstellungen noch akzeptabel - , und er erlebt seinen tiefen Fall durch seinen „tragic flaw“ (hier seine tragische Charakterschwäche), aber er ist auch ein vielfacher Mörder und stellt sich dadurch gegen die hehre Auffassung von Tragik. Demnach hat er seine Bestrafung reichlich verdient, nicht jedoch „fear and pity“.

\section{Ausblick}

In einer Klausuraufgabe wurden Schüler nach ihrem Urteil über Macbeth gefragt, wenn dieser am Ende überlebt hätte und vor Gericht gestellt worden wäre. Die Mehrheit billigte ihm mildernde Umstände zu bzw. versuchte, ihn zu entschuldigen und geriet damit sogar in die Nähe einer Rechtfertigung seiner Taten. Die Begründung lautete: er sei doch zweimal verführt worden, einmal durch die Hexen, zum anderen durch Lady Macbeth. An keiner Stelle wurde die Wirkung von Sympathielenkung so deutlich wie hier.

Daher erweisen sich im Sinne dessen, was die einleitenden Beispiele aus dem Schulbuch und Hemingways Roman wie auch die Erörterung der Füssli-Bilder aussagen sollten, erst recht im Zusammenhang rnit Macbeth klärende Gespräche über die Wirkung von Sympathielenkung als notwendig. Sympathielenkung kann sich als simple Leser- und Zuschauerverführung entwickeln und differenzierende Betrachtungsweisen verhindern. Dann bliebe weitgehend unberücksichtigt, was im Denken und Handeln der Personen moralisch-ethisch und rechtlich angemessen bzw. nicht angemessen ist.

Daher sollten Schüler im Literaturunterricht, auf welchem methodischdidaktischen Weg auch immer, dazu befähigt werden, dass sie mit einem Text differenzierend und letztlich auch kritisch umgehen können. Bildung ist auch immer ein Stück Lebenshilfe, und daher sollte der Literaturunterricht in den Schulen wie auch die Beschäftigung mit Literatur im Privaten als eine unverzichtbare Bereicherung des menschlichen Seins betrachtet werden. 


\section{Verwendete Literatur}

Primärwerke

Garner, Hugh. „The Moose and the Sparrow“. Learning English - Modern Course Gym 6. Hg. Werner Beile u.a.. Stuttgart: Ernst Klett, 1982. 14-15.

Hemingway, Ernest. A Farewell to Arms. London: Penguin, 1965.

Russel, Willy. Educating Rita. Ed. and annotated by A.-R. Glaap. Frankfurt a.M.: Diesterweg, 1997.

Shakespeare, William. Macbeth. Ed. by Kenneth Muir. London: Arden, 2002.

---. Macbeth. Zweisprachige Ausgabe. Deutsch von Frank Günther. München: Deutscher Taschenbuch Verlag, 2006.

---. Macbeth. Englisch und Deutsch. Hg. L. L. Schücking. Übersetzung August Wilhelm Schlegel und Ludwig Tieck. Berlin und Darmstadt: Rowohlt, 1963.

---. Richard III. Englisch und Deutsch. Hg. L. L. Schücking. Übersetzung August Wilhelm Schlegel und Ludwig Tieck. Berlin und Darmstadt: Rowohlt, 1958.

Sekundärwerke

Pape, Walter und Frederick Burwick, Hg. The Boydell Shakespeare Gallery. Bottrop: Pomp, 1996.

Pfister, Manfred. „Zur Theorie der Sympathielenkung im Drama.“ Sympathielenkung in den Dramen Shakespeares. Studien zur publikumsbezogenen Dramaturgie. Hg. Werner Habicht, Ina Schabert. München: Wilhelm Fink, 1978. 20-34.

Schabert, Ina. „Sympathy“ als rezeptionsästhetische Kategorie in der englischen und amerikanischen Shakespearekritik". Sympathielenkung in den Dramen Shakespeares. Studien zur publikumsbezogenen Dramaturgie. Hg. Werner Habicht, Ina Schabert. München: Wilhelm Fink, 1978. 35-53.

Video

TV-Aufzeichnung: Macbeth by Lincoln Center Theater Company. New York, 1981. Philip Anglim as Macbeth, Maureen Anderman as Lady Macbeth. Directed by Kirk Browning. 